\title{
Temozolomide for children and adolescents with diffuse intrinsic pontine glioma (Protocol)
}

Jansen MHA, Veringa SJE, van Vuurden DG, van Dalen EC, Kaspers GJL
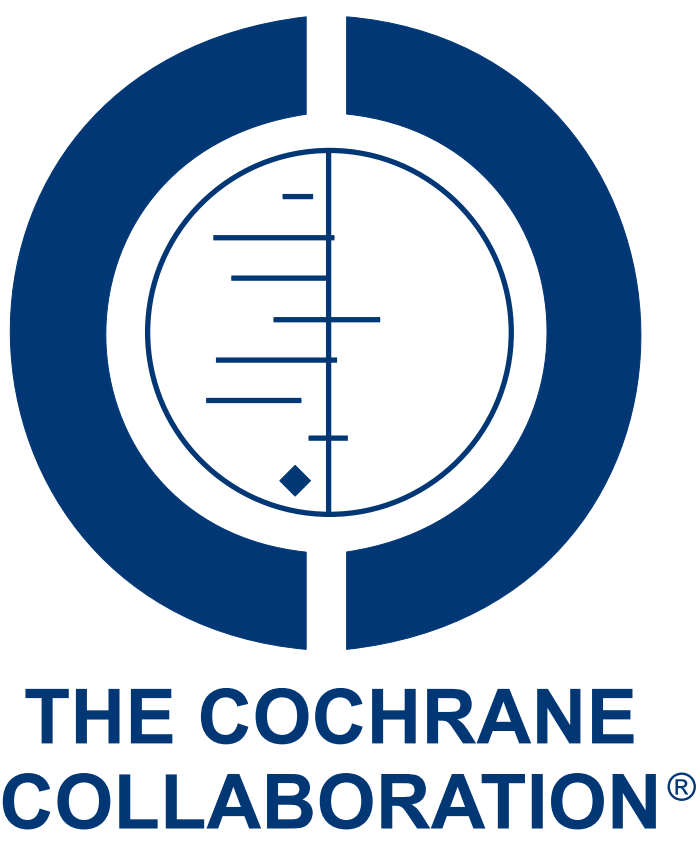

This is a reprint of a Cochrane protocol, prepared and maintained by The Cochrane Collaboration and published in The Cochrane Library 2012, Issue 10

http://www.thecochranelibrary.com

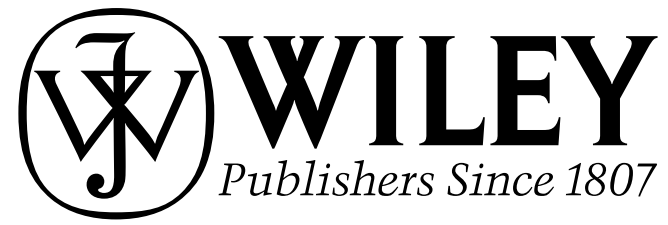

Temozolomide for children and adolescents with diffuse intrinsic pontine glioma (Protocol)

Copyright $\odot 2012$ The Cochrane Collaboration. Published by John Wiley \& Sons, Ltd. 
TABLE OF CONTENTS

HEADER . . . . . . . . . . . . . . . . . . . . . . . . . . . . . . . . . . . . . . . . 1

ABSTRACT . . . . . . . . . . . . . . . . . . . . . . . . . . . . . . . . . . . . . . 1

BACKGROUND . . . . . . . . . . . . . . . . . . . . . . . . . . . . . . . . . . . . . 2

OBJECTIVES . . . . . . . . . . . . . . . . . . . . . . . . . . . . . . . . . . . . . . . .

METHODS . . . . . . . . . . . . . . . . . . . . . . . . . . . . . . . . . . . . . .

ACKNOWLEDGEMENTS . . . . . . . . . . . . . . . . . . . . . . . . . . . . . . . . . . . . . . .

REFERENCES . . . . . . . . . . . . . . . . . . . . . . . . . . . . . . . . . . . . . . 5

ADDITIONAL TABLES . . . . . . . . . . . . . . . . . . . . . . . . . . . . . . . . . . . . . . . . 7

APPENDICES . . . . . . . . . . . . . . . . . . . . . . . . . . . . . . . . . . . . . 8

HISTORY . . . . . . . . . . . . . . . . . . . . . . . . . . . . . . . . . . . . . . . . 10

DECLARATIONS OF INTEREST . . . . . . . . . . . . . . . . . . . . . . . . . . . . . . . . . . . . .

SOURCES OF SUPPORT . . . . . . . . . . . . . . . . . . . . . . . . . . . . . . . . . . . .

Temozolomide for children and adolescents with diffuse intrinsic pontine glioma (Protocol)

Copyright $\odot 2012$ The Cochrane Collaboration. Published by John Wiley \& Sons, Ltd. 


\title{
[Intervention Protocol]
}

\section{Temozolomide for children and adolescents with diffuse intrinsic pontine glioma}

\author{
Marc HA Jansen $^{1}$, Susanna JE Veringa ${ }^{1}$, Dannis G van Vuurden ${ }^{1}$, Elvira C van Dalen ${ }^{2}$, Gertjan JL Kaspers ${ }^{1}$ \\ ${ }^{1}$ Department of Paediatrics, Division of Paediatric Oncology/Haematology, VU University Medical Center, Amsterdam, Netherlands. \\ ${ }^{2}$ Department of Paediatric Oncology, Emma Children’s Hospital / Academic Medical Center, Amsterdam, Netherlands \\ Contact address: Marc HA Jansen, Department of Paediatrics, Division of Paediatric Oncology/Haematology, VU University Medical \\ Center, PO Box 7057, Amsterdam, 1007 MB, Netherlands. mh.jansen@vumc.nl.
}

Editorial group: Cochrane Childhood Cancer Group.

Publication status and date: New, published in Issue 10, 2012.

Citation: Jansen MHA, Veringa SJE, van Vuurden DG, van Dalen EC, Kaspers GJL. Temozolomide for children and adolescents with diffuse intrinsic pontine glioma. Cochrane Database of Systematic Reviews 2012, Issue 10. Art. No.: CD010129. DOI: 10.1002/14651858.CD010129.

Copyright (C) 2012 The Cochrane Collaboration. Published by John Wiley \& Sons, Ltd.

\section{A B S T R A C T}

This is the protocol for a review and there is no abstract. The objectives are as follows:

To assess the efficacy of temozolomide in children and adolescents with newly diagnosed and progressive DIPG. 


\section{B A C K G R O U N D}

\section{Description of the condition}

Diffuse intrinsic pontine glioma (DIPG) is one of the most devastating childhood cancers. The incidence is 0.1 per 100,000 children per year and DIPG accounts for 7 percent of all pediatric central nervous system tumors (Kaatsch 2001). DIPG usually concerns high grade glioma (grade III or glioblastoma multiforme) but can also be grade II diffuse astrocytoma (Fisher 2000); even primitive neuroectodermal tumor (PNET) histology has been described in occasional subjects (Zarghooni 2010). Diagnosis is based on magnetic resonance imaging (MRI), which typically shows a diffusely swollen pons and encasement of the basilary artery by the tumor. Treatment is difficult because of the delicate location within the pons from which the majority of the cranial nerves originate. This makes radical surgery too dangerous (Epstein 1986). In addition, several anti-cancer drugs appeared to be inactive in DIPG, either due to tumor drug resistance and/or poor drug distribution in the tumor due to a frequently intact blood brain barrier (Hargrave 2006; Hargrave 2008). Radiotherapy can significantly reduce the tumor and often improves clinical signs, but these effects are almost always temporary (Langmoen 1991). For these reasons, the prognosis of DIPG has remained unchanged in the past 30 years with a median overall survival rate of nine months and a twoyear overall survival rate of less than 10 percent ( Hargrave 2006; Jansen 2012).

\section{Description of the intervention}

Temozolomide, an imidazotetrazine derivative of dacarbazine, is an oral alkylating drug. Once temozolomide enters the systemic circulation it is converted to its active compound, monomethyl triazeno imidazole carboxamide (MTIC) (Stevens 1987).

Nowadays, the use of temozolomide in the treatment of pediatric and adult glioma is widespread. This extensive use resulted from the report of a phase III trial in 2005, in which temozolomide was given concurrently with radiotherapy and as adjuvant treatment for adult high grade glioma (Stupp 2005). The median overall survival increased from 12.1 to 14.6 months $(\mathrm{P}<0.001)$ with temozolomide compared to radiotherapy alone. This study resulted in the approval of temozolomide for the treatment of newly diagnosed glioblastoma multiforme (GBM). These results were confirmed in a systematic review on temozolomide for high grade glioma in 2008 (Hart 2008). In addition, temozolomide was also approved for the treatment of refractory anaplastic astrocytoma. In the mean time, phase I studies with temozolomide in children were initiated. Temozolomide was found to be safe in children with mainly hematological toxicity, which was comparable to that been observed in adults (Estlin 1998).

\section{Why it is important to do this review}

After the successes obtained in adults, multiple temozolomide trials were initiated in children and young adults with glioma, both with conventional and protracted dose schedules (Baruchel 2006; Broniscer 2006; Cohen 2011b; Jakacki 2008; Lashford 2002; Nicholson 2007; Ruggiero 2006; Verschuur 2004). This also applies to DIPG, where multiple studies were published from 2005 with temozolomide alone or in combination with other drugs (Broniscer 2005; Chassot 2012; Cohen 2011a; Jalali 2009; Kim 2010; Sharp 2010; Sirachainan 2008). Furthermore, temozolomide became a drug that was frequently prescribed off protocol to patients with DIPG. However, the efficacy of temozolomide in children with DIPG is unclear. To the best of our knowledge, no systematic review has been done on temozolomide in children and adolescents with DIPG thus far. The results from this systematic review thus answer the clinical question whether children and adolescents with DIPG should or should not be treated with temozolomide and to identify subgroups of patients who may benefit from temozolomide treatment.

\section{O B J E C T I V E S}

To assess the efficacy of temozolomide in children and adolescents with newly diagnosed and progressive DIPG.

\section{METHODS}

\section{Criteria for considering studies for this review}

\section{Types of studies}

We will include randomized controlled trials (RCTs), clinically controlled trials (CCTs), unrandomized single-arm trials with historical controls and cohort studies examining the efficacy of temozolomide in the treatment of childhood and adolescent DIPG. Cross-sectional studies, case-reports and case series (i.e. a series of non-consecutive patients) will be excluded. A cohort study is defined as a study in which a group of consecutive patients treated for DIPG was followed either from diagnosis or from time of progression onwards. The described study could be the original cohort or a subgroup of the original cohort based on well-defined inclusion criteria. Studies including less than five patients will be excluded.

\section{Types of participants}

Patients aged $<21$ years with clinical and MRI features consistent with the diagnosis of diffuse intrinsic pontine glioma are eligible. 
Patients can be either newly diagnosed with no prior therapy other than corticosteroids or with disease progression after radiotherapy. The MRI criteria include a T1 iso- or hypointense, T2 hyperintense tumor originating in the pons, with at least $50 \%$ involvement of the pontine region. Patients diagnosed by computed tomography (CT) only will be excluded, since CT is not sensitive for diagnosing pontine glioma (Packer 1985). Histological confirmation by means of biopsy is not mandatory. In cases where pathologic analysis of the tumor is available, histological diagnosis should be grade 2-4 astrocytoma or grade 2-3 oligodendroglioma or oligoastrocytoma, located in the pons (Fisher 2000). We will exclude exophytic tumors and cases of pilocytic astrocytomas and primitive neuroectodermal tumors (PNET) of the brainstem. If studies include both eligible and non-eligible patients, the results of only eligible patients should be available for inclusion in the review.

\section{Types of interventions}

Treatment with temozolomide, either neo-adjuvant, concomitant or adjuvant to fractionated radiotherapy. Both treatment arms of the RCT or CCT or, in case the control group is a historical cohort, both the current and historical cohort, should receive fractionated radiotherapy. Treatment protocols are allowed to include supportive care, e.g. glucocorticosteroids, anti-epileptics and other drugs depending on appropriate clinical need, as well as additional anticancer drugs in the treatment regimen.

\section{Types of outcome measures}

\section{Primary outcomes}

\section{Newly diagnosed patients}

- Overall survival, defined as the interval between tumor diagnosis and death from any cause.

\section{Patients with progressive disease}

- Radiological response rate, defined as the total number of patients with a complete or partial response. Response criteria as assessed by MRI according to definitions of the authors of the original study.

\section{Secondary outcomes}

\section{Newly diagnosed patients}

- Progression free survival, defined as the interval between tumor diagnosis and clinical and/or radiological tumor progression or death
- Radiological response evaluation, defined as the total number of patients with a complete or partial response. Response criteria as assessed by MRI according to definitions of the authors of the original study

\section{Patients with progressive disease}

- Overall survival defined as the interval between start of temozolomide treatment and death

- Progression free survival defined as the interval between start of temozolomide treatment and clinical and/or radiological tumor progression or death

\section{Both groups}

- Clinical response in terms of reduction in signs or symptoms(endpoint: total number of clinical responses)

- Adverse events as defined by the authors of the original study

- Performance score based on Karnofsky 1949 or Lansky 1987

\section{Search methods for identification of studies}

We will use the Cochrane Childhood Cancer Group search methods (Kremer 2011). We will not impose language restrictions. Searches will be updated every two years.

\section{Electronic searches}

We will search the following electronic databases: the Cochrane Central Register of Controlled Trials (CENTRAL) in The Cochrane Library (latest issue), MEDLINE (PubMed; from 1945 to present) and EMBASE (Ovid; from 1980 to present).

The search strategies for the different electronic databases (using a combination of controlled vocabulary and text words) are shown in the appendices (Appendix 1; Appendix 2; Appendix 3)

\section{Searching other resources}

We will locate information on trials not registered in MEDLINE, EMBASE or CENTRAL, either published or unpublished, by searching the reference lists of relevant articles and review articles. We will handsearch the conference proceedings of the International Society for Paediatric Oncology (SIOP), the Society for Pediatric Neuro-Oncology (SPNO), the Society for NeuroOncology (SNO), the American Association for Cancer Research (AACR), the American Society of Clinical Oncology (ASCO), the European Association for Cancer Research (EACR), and the European Association for Neuro-Oncology (EANO) published between 2006 and 2011. We will scan the World Health Organization International Clinical Trials Registry Platform (ICTRP) search portal (http://apps.who.int/trialsearch/), the International 
Standard Randomised Controlled Trial Number Register (ISRCTN Register) (http://www.controlled-trials.com/isrctn/) and ClinicalTrials.gov (http://clinicaltrials.gov/) for ongoing trials.

\section{Data collection and analysis}

\section{Selection of studies}

After performing the search strategy described previously, two authors (MJ, SV) will independently select studies meeting the inclusion criteria. All discrepancies between the two authors will be resolved by consensus. Otherwise, final resolution will be achieved by using a third-party arbitrator (DvV, GJK, EVD). We will obtain in full any study which seems to meet the inclusion criteria on grounds of the title and/or abstract for closer inspection. We will clearly state details of reasons for exclusion of any study considered for this review.

\section{Data extraction and management}

Two authors (MJ, SV) will independently perform data extraction using standardized forms. Discrepancies between authors will be resolved by discussion. If this is impossible, we will achieve final resolution using a third-party arbitrator (DvV, GJK, EVD).

Data will be extracted on the following items:

1. Study characteristics

- Design

- Number of patients enrolled in the study

- Number of patients fulfilling the authors' inclusion criteria

2. Participants characteristics

- Sex

- Age at time of diagnosis

- Duration of symptoms at diagnosis

- MRI characteristics

- Stage of disease

- Biopsy for histology and $\mathrm{O}^{6}$-methylguanine-DNA methyltransferase (MGMT) promoter methylation status

- Neurofibromatosis status

3. Interventions

- Cumulative radiation dose (Gy) and number of fractions

- Temozolomide treatment protocol (dose and schedule)

- Neo-adjuvant, concomitant and/or adjuvant scheme

- Additive therapeutics (dose and schedule)

4. Outcome measures

- Overall survival (months)

- Progression free survival (months)

- Radiological response evaluation

- Clinical response

- Adverse events

- Performance scores

\section{Length of follow-up}

\section{Assessment of risk of bias in included studies}

Two review authors will independently perform the assessment of risk of bias of the included studies (MJ and SV). For RCTs and CCTs we will use the risk of bias items as described in the module of the Childhood Cancer Group (Kremer 2011), which are based on the Cochrane Handbook for Systematic Reviews of Interventions (Higgins 2011). The assessment of risk of bias in observational studies will be based on previously described checklists according to Evidence-Based Medicine Criteria (Grimes 2002; Laupacis 1994). See Table 1 for the definitions of the different risk of bias criteria. The risk of bias in included studies will be taken into account in the interpretation of the review's results. We will resolve discrepancies between authors by consensus. If this is not possible, we will achieve final resolution using a third-party arbitrator (DvV, GJK, EVD).

\section{Measures of treatment effect}

If a control group is available, dichotomous variables will be analyzed using risk ratios (RRs); continuous outcomes will be analyzed using mean differences (MDs), survival will be analyzed using hazard ratios (HRs). We will use Parmar's method if HRs were not explicitly presented in the study (Parmar 1998). If no control group is available, we will use the prevalence to analyze radiological response and adverse effects; survival will be summarized descriptively. All results will be presented with the corresponding $95 \%$ confidence intervals (CIs).

\section{Dealing with missing data}

When relevant data regarding study selection, data extraction and risk of bias assessment are missing, we will attempt to contact the study authors to retrieve the missing data. We will extract data by the allocated intervention, irrespective of compliance with the allocated intervention, in order to allow an intention-to-treat analysis. If this is not possible, this will be stated and we will perform a per-protocol analysis.

\section{Assessment of heterogeneity}

We will assess heterogeneity both by visual inspection of the forest plots and by a formal statistical test for heterogeneity, the I 2 statistic. In the absence of significant heterogeneity $\left(\mathrm{I}^{2}<50 \%\right)$ (Higgins 2011), we will use a fixed-effect model for the estimation of treatment effects. Otherwise, we will explore possible reasons for the occurrence of heterogeneity and take appropriate measures. 


\section{Assessment of reporting biases}

In addition to the evaluation of reporting bias as described in the 'Assessment of risk of bias in included studies' section, we will assess reporting bias by constructing a funnel plot if at least 10 studies are included in a meta-analysis. When there are fewer studies the power of the tests is too low to distinguish chance from real asymmetry (Higgins 2011).

\section{Data synthesis}

We will enter data into the Cochrane Collaboration's statistical software, Review Manager (Review Manager 2011), and undertake analyses according to the guidelines of the Cochrane Handbook for Systematic Reviews of Interventions (Higgins 2011). We will include outcome measures only if it was the intention of the study to perform the necessary assessments in all patients (that is, not only optional or only performed in some centers). Newly diagnosed patients and patients with progressive disease will be analyzed separately. We will pool results only if studies are comparable with regard to important prognostic factors (e.g. percentage of pontine tumor infiltration, time between onset of symptoms and diagnosis and age at diagnosis) and used outcome definitions. Different study designs will be taken into account in the analyses. Studies for which pooling of results is not possible will be summarized descriptively.

\section{Subgroup analysis and investigation of heterogeneity}

The following variables may influence prognosis:

- newly diagnosed versus progressive DIPG;

- age $\geq$ three years versus $<$ three years at time of diagnosis: younger age correlates with a better prognosis (Broniscer 2008);

- time between onset of symptoms (more or less than six months) and diagnosis: shorter duration of symptoms may reflect more aggressive tumors with a worse prognosis (Ueoka 2009)
- histology: low grade versus high grade glioma; the latter may reflect a slightly worse prognosis (Wagner 2006);

- neurofibromatosis type 1 (NF-1): an increased survival among DIPG patients considered to have NF-1 has been reported (Milstein 1989);

- percentage of pontine infiltration by the tumor;

- enhancement on MRI at diagnosis or enhancement that correlates with a worse overall survival (Poussaint 2011);

- $\mathrm{O}^{6}$-methylguanine-DNA methyltransferase (MGMT) promoter methylation status of the tumor; MGMT methylation correlates with an increased response to temozolomide (Hegi 2005); and

- additional, second- or third-line therapeutics.

\section{Sensitivity analysis}

For all outcomes for which pooling is possible we will perform sensitivity analyses for all risk of bias criteria separately. We will exclude studies of high risk of bias and studies for which the risk of bias is unclear and compare the results of studies with a low risk of bias with the results of all available studies.

\section{ACKNOW LEDGEMENTS}

We thank Edith Leclercq for her help with developing the search strategies for the different databases.

The editorial base of the Cochrane Childhood Cancer Group is funded by Stichting Kinderen Kankervrij (KiKa).

We thank the following referees for their insightful and constructive comments: U. Bartels, MD, R. Jalali, MD, and G. Dhall, MD.

\section{REFERE N CES}

\section{Additional references}

\section{Baruchel 2006}

Baruchel S, Diezi M, Hargrave D, Stempak D, Gammon

$\mathrm{J}$, Moghrabi A, et al.Safety and pharmacokinetics of temozolomide using a dose-escalation, metronomic schedule in recurrent paediatric brain tumours. European Journal of Cancer 2006;42(14):2335-42.

Broniscer 2005

Broniscer A, Iacono L, Chintagumpala M, Fouladi M, Wallace D, Bowers DC, et al.Role of temozolomide after radiotherapy for newly diagnosed diffuse brainstem glioma in children: results of a multiinstitutional study (SJHG-98). Cancer 2005;103(1):133-9.

\section{Broniscer 2006}

Broniscer A, Chintagumpala M, Fouladi M, Krasin MJ, Kocak M, Bowers DC, et al.Temozolomide after radiotherapy for newly diagnosed high-grade glioma and unfavorable low-grade glioma in children. Journal of Neurooncology 2006;76(3):313-9.

\section{Broniscer 2008}

Broniscer A, Laningham FH, Sanders RP, Kun LE, Ellison DW, Gajjar A. Young age may predict a better outcome for children with diffuse pontine glioma. Cancer 2008;113(3): 566-72.

\section{Chassot 2012}

Chassot A, Canale S, Varlet P, Puget S, Roujeau T, Negretti L, et al.Radiotherapy with concurrent and adjuvant 
temozolomide in children with newly diagnosed diffuse intrinsic pontine glioma. Journal of Neurooncology 2012; 106(2):399-407.

\section{Cohen 2011a}

Cohen KJ, Heideman RL, Zhou T, Holmes EJ, Lavey RS, Bouffet E, et al.Temozolomide in the treatment of children with newly diagnosed diffuse intrinsic pontine gliomas: a report from the Children's Oncology Group. Neurooncology 2011;13(4):410-6.

\section{Cohen 2011b}

Cohen KJ, Pollack IF, Zhou T, Buxton A, Holmes EJ, Burger PC, et al.Temozolomide in the treatment of highgrade gliomas in children: a report from the Children's Oncology Group. Neurooncology 2011;13(3):317-23.

\section{Epstein 1986}

Epstein F, McCleary EL. Intrinsic brain-stem tumors of childhood: surgical indications. Journal of Neurosurgery 1986;64(1):11-5.

\section{Estlin 1998}

Estlin EJ, Lashford L, Ablett S, Price L, Gowing R, Gholkar A, et al.Phase I study of temozolomide in paediatric patients with advanced cancer. United Kingdom Children's Cancer Study Group. British Journal of Cancer 1998;78(5):652-61.

Fisher 2000

Fisher PG, Breiter SN, Carson BS, Wharam MD, Williams JA, Weingart JD, et al.A clinicopathologic reappraisal of brain stem tumor classification. Identification of pilocystic astrocytoma and fibrillary astrocytoma as distinct entities. Cancer 2000;89(7):1569-76.

\section{Grimes 2002}

Grimes DA, Schulz KF. Cohort studies: marching towards outcomes. Lancet 2002;359(9303):341-5.

\section{Hargrave 2006}

Hargrave D, Bartels U, Bouffet E. Diffuse brainstem glioma in children: critical review of clinical trials. Lancet Oncology 2006;7(3):241-8.

\section{Hargrave 2008}

Hargrave D, Chuang N, Bouffet E. Conventional MRI cannot predict survival in childhood diffuse intrinsic pontine glioma. Journal of Neurooncology 2008;86(3): $313-9$.

\section{Hart 2008}

Hart MG, Grant R, Garside R, Rogers G, Somerville M, Stein K. Temozolomide for High Grade Glioma. Cochrane Database of Systematic Reviews 2008, Issue 4. [DOI: 10.1002/14651858.CD007415]

Hegi 2005

Hegi ME, Diserens AC, Gorlia T, Hamou MF, de Tribolet $\mathrm{N}$, Weller M, et al.MGMT gene silencing and benefit from temozolomide in glioblastoma. New England Journal of Medicine 2005;352(10):997-1003.

Higgins 2011

Higgins JPT, Green S (editors). Higgins JPT, Green $S$ (editors). Cochrane Handbook for Systematic Reviews of Interventions Version 5.1.0 [updated March 2011].
The Cochrane Collaboration, 2011. Available from www.cochrane-handbook.org.

\section{Jakacki 2008}

Jakacki RI, Hamilton M, Gilbertson RJ, Blaney SM, Tersak J, Krailo MD, et al.Pediatric phase I and pharmacokinetic study of erlotinib followed by the combination of erlotinib and temozolomide: a Children's Oncology Group Phase I Consortium Study. Journal of Clinical Oncology 2008;26 (30):4921-7.

\section{Jalali 2009}

Jalali R, Raut N, Arora B, Gupta T, Dutta D, Munshi A, et al.Prospective evaluation of radiotherapy with concurrent and adjuvant temozolomide in children with newly diagnosed diffuse intrinsic pontine glioma. International Journal of Radiation Oncology ${ }^{*}$ Biology ${ }^{*}$ Physics 2009;77(1): 113-8.

Jansen 2012

Jansen MH, van Vuurden DG, Vandertop WP, Kaspers GJ. Diffuse intrinsic pontine gliomas: A systematic update on clinical trials and biology. Cancer Treatment Reviews 2012; 38(1):27-35.

\section{Kaatsch 2001}

Kaatsch P, Rickert CH, Kuhl J, Schuz J, Michaelis J. Population-based epidemiologic data on brain tumors in German children. Cancer 2001;92(12):3155-64.

\section{Karnofsky 1949}

Karnofsky DA, Burchenal JH. The Clinical Evaluation of Chemotherapeutic Agents in Cancer. MacLeod CM (Ed), Evaluation of Chemotherapeutic Agents. Columbia Univ Press, 1949:196.

\section{Kim 2010}

Kim CY, Kim SK, Phi JH, Lee MM, Kim IA, Kim IH, et al.A prospective study of temozolomide plus thalidomide during and after radiation therapy for pediatric diffuse pontine gliomas: preliminary results of the Korean Society for Pediatric Neuro-Oncology study. Journal of Neurooncology 2010;100(2):193-8.

\section{Kremer 2011}

Kremer LCM, van Dalen EC, Moher D, Caron HN. Cochrane Childhood Cancer Group. The Cochrane Library 2011, issue 12 .

\section{Langmoen 1991}

Langmoen IA, Lundar T, Storm-Mathisen I, Lie SO, Hovind KH. Management of pediatric pontine gliomas. Child's Nervous System 1991;7(1):13-5.

\section{Lansky 1987}

Lansky SB, List MA, Lansky LL, Ritter-Sterr C, Miller DR. The measurement of performance in childhood cancer patients. Cancer 1987;60(7):1651-6.

\section{Lashford 2002}

Lashford LS, Thiesse P, Jouvet A, Jaspan T, Couanet D, Griffiths $\mathrm{PD}$, et al.Temozolomide in malignant gliomas of childhood: a United Kingdom Children's Cancer Study Group and French Society for Pediatric Oncology 
Intergroup Study. Journal of Clinical Oncology 2002;20(24): 4684-91.

\section{Laupacis 1994}

Laupacis A, Wells G, Richardson WS, Tugwell P. Users' guides to the medical literature. V. How to use an article about prognosis. Evidence-Based Medicine Working Group. JAMA 1994;272(3):234-7.

Milstein 1989

Milstein JM, Geyer JR, Berger MS, Bleyer WA. Favorable prognosis for brain stem gliomas in neurofibromatosis. Journal of Neurooncology 1989;7:367-71.

Nicholson 2007

Nicholson HS, Kretschmar CS, Krailo M, Bernstein M, Kadota R, Fort D, et al.Phase 2 study of temozolomide in children and adolescents with recurrent central nervous system tumors: a report from the Children's Oncology Group. Cancer 2007;110(7):1542-50.

\section{Packer 1985}

Packer RJ, Zimmerman RA, Luerssen TG, Sutton LN, Bilaniuk LT, Bruce DA, et al.Brainstem gliomas of childhood: magnetic resonance imaging. Neurology 1985; 35(3):397-401.

\section{Parmar 1998}

Parmar MK, Torri V, Stewart L. Extracting summary statistics to perform meta-analyses of the published literature for survival endpoints. Statistics Medical 1998;17(24): 2815-34.

Poussaint 2011

Poussaint TY, Kocak M, Vajapeyam S, Packer RI, Robertson RL, Geyer R, et al.MRI as a central component of clinical trials analysis in brainstem glioma: a report from the Pediatric Brain Tumor Consortium (PBTC). Neurooncology 2011;13(4):417-27.

\section{Review Manager 2011}

The Nordic Cochrane Centre, The Cochrane Collaboration. Review Manager (RevMan). 5.1. Copenhagen: The Nordic Cochrane Centre, The Cochrane Collaboration, 2011.

Ruggiero 2006

Ruggiero A, Cefalo G, Garre ML, Massimino M, Colosimo $\mathrm{C}$, Attina G, et al.Phase II trial of temozolomide in children with recurrent high-grade glioma. Journal of Neurooncology 2006;77(1):89-94.

\section{Sharp 2010}

Sharp JR, Bouffet E, Stempak D, Gammon J, Stephens D, Johnston DL, et al.A multi-centre Canadian pilot study of metronomic temozolomide combined with radiotherapy for newly diagnosed paediatric brainstem glioma. European Journal of Cancer 2010;46(18):3271-9.

Sirachainan 2008

Sirachainan N, Pakakasama S, Visudithbhan A, Chiamchanya $S$, Tuntiyatorn L, Dhanachai M, et al.Concurrent radiotherapy with temozolomide followed by adjuvant temozolomide and cis-retinoic acid in children with diffuse intrinsic pontine glioma. Neurooncology 2008; 10(4):577-82.

\section{Stevens 1987}

Stevens MF, Hickman JA, Langdon SP, Chubb D, Vickers $\mathrm{L}$, Stone R, et al.Antitumor activity and pharmacokinetics in mice of 8-carbamoyl-3-methyl-imidazo[5,1-d]-1,2,3,5tetrazin-4(3H)-one (CCRG 81045; M \& B 39831), a novel drug with potential as an alternative to dacarbazine. Cancer Research 1987;47(22):5846-52.

\section{Stupp 2005}

Stupp R, Mason WP, van den Bent MJ, Weller M, Fisher B, Taphoorn MJ, et al.Radiotherapy plus concomitant and adjuvant temozolomide for glioblastoma. New England Journal of Medicine 2005; Vol. 352, issue 10:987-96.

Ueoka 2009

Ueoka DI, Nogueira J, Campos JC, Maranhão Filho P, Ferman S, Lima MA. Brainstem gliomas-retrospective analysis of 86 patients. Journal of Neurological Sciences 2009; 281(1-2):20-3.

Verschuur 2004

Verschuur AC, Grill J, Lelouch-Tubiana A, Couanet D, Kalifa C, Vassal G. Temozolomide in paediatric high-grade glioma: a key for combination therapy?. British Journal of Cancer 2004;91(3):425-9.

\section{Wagner 2006}

Wagner S, Warmuth-Metz M, Emser A, Gnekow AK, Strater R, Rutkowski S, et al.Treatment options in childhood pontine gliomas. Journal of Neurooncology 2006;79(3): 281-7.

\section{Zarghooni 2010}

Zarghooni M, Bartels U, Lee E, Buczkowicz P, Morrison A, Huang A, et al.Whole-genome profiling of pediatric diffuse intrinsic pontine gliomas highlights platelet-derived growth factor receptor alpha and poly (ADP-ribose) polymerase as potential therapeutic targets. Journal of Clinical Oncology 2010;28(8):1337-44.

* Indicates the major publication for the study 
ADDITIONAL TABLES

Table 1. Risk of bias criteria for observational studies

\begin{tabular}{|c|c|c|}
\hline & Internal validity & External validity \\
\hline Study group & $\begin{array}{l}\text { Selection bias (representative: yes/no): } \\
\text { - If the described study group consisted of more than } \\
90 \% \text { of the DIPG patients treated with temozolomide } \\
\text { included in the original cohort } \\
\text { - Or if it was a random sample of these patients with } \\
\text { respect to important prognostic factors (like percentage } \\
\text { of pontine tumor infiltration, time between onset of } \\
\text { symptoms and diagnosis and age at diagnosis) and } \\
\text { cancer treatment }\end{array}$ & $\begin{array}{l}\text { Reporting bias (well defined: yes/no): } \\
\text { - If the mean, median or range of the cumulative } \\
\text { temozolomide dose was mentioned } \\
\text { - And when it was described what other treatment } \\
\text { (including the received doses) was given } \\
\text { - And when the MRI criteria used for tumor } \\
\text { diagnosis were mentioned } \\
\text { - And when the mean, median or range of the } \\
\text { patients age was mentioned }\end{array}$ \\
\hline Follow-up & $\begin{array}{l}\text { Attrition bias (adequate: yes/no): } \\
\text { - If the outcome was assessed for more than } 90 \% \text { of } \\
\text { the study group of interest }(++) \\
\text { - Or if the outcome was assessed for } 60-90 \% \text { of the } \\
\text { study group of interest }(+)\end{array}$ & $\begin{array}{l}\text { Reporting bias (well-defined: yes/no): } \\
\text { - If the length of follow-up was mentioned }\end{array}$ \\
\hline Outcome & $\begin{array}{l}\text { Detection bias (blind: yes/no): } \\
\text { - If the outcome assessors were blinded to the } \\
\text { investigated determinant }\end{array}$ & $\begin{array}{l}\text { Reporting bias (well-defined: yes/no): } \\
\text { - If the outcome definition was provided }\end{array}$ \\
\hline
\end{tabular}

DIPG: diffuse intrinsic pontine glioma

\section{A P P E N D I C E S}

\section{Appendix I. Search strategy for CENTRAL (The Cochrane Library)}

1. For Temolozomide the following text words will be used:

Temolozomide OR 8-carbamoyl-3-methylimidazo(5,1-d)-1,2,3,5-tetrazin-4(3H)-one OR methazolastone OR MB 39831 OR MB39831 OR Temodar OR Temodal OR Schering-Plough brand of temozolomide OR Essex brand of temozolomide OR Schering brand of temozolomide OR TMZ-Bioshuttle OR CCRG 81045 OR CCRG-81045 OR NSC 362856 OR NSC-362856 OR 85622-93-1

2. For Gliomas the following text words will be used:

diffuse intrinsic pontine glioma OR diffuse intrinsic pontine gliomas OR diffuse intrinsic pontine gliom* OR DIPG OR pontine gliom* OR diffuse pontine gliom* OR brainstem gliom* OR brain stem gliom* OR brainstem tumor* OR brainstem tumour* OR brain stem tumor* OR brain stem tumour* OR high grade glioma OR high grade gliomas OR high grade gliom* OR glioblastoma multiforme OR glioblastoma OR glioblastom* OR anaplastic astrocytoma OR fibrillary astrocytoma OR GBM OR diffuse astrocytoma OR diffuse fibrillary astrocytoma OR astrocytom*

3. For Chilldren and adolescents the following text words will be used:

infan* OR newborn* OR new-born* OR perinat* OR neonat* OR baby OR baby* OR babies OR toddler* OR minors OR minors* OR boy OR boys OR boyfriend OR boyhood OR girl* OR kid OR kids OR child OR child* OR children* OR schoolchild* OR schoolchild OR school child[tiab] OR school child*[tiab] OR adolescen* OR juvenil* OR youth* OR teen* OR under*age* OR

Temozolomide for children and adolescents with diffuse intrinsic pontine glioma (Protocol)

Copyright @ 2012 The Cochrane Collaboration. Published by John Wiley \& Sons, Ltd. 
pubescen* OR pediatrics OR pediatric* OR paediatric* OR peadiatric* OR school OR school* OR prematur* OR preterm* OR "young adult" OR "young adults"

Final search 1 AND 2 AND 3

The search will be performed in title, abstract or keywords

$\left[{ }^{*}=\right.$ zero or more characters $]$

\section{Appendix 2. Search strategy for MEDLINE (PubMed)}

1. For Temolozomide the following MeSH headings and text words will be used:

Temolozomide OR 8-carbamoyl-3-methylimidazo(5,1-d)-1,2,3,5-tetrazin-4(3H)-one OR methazolastone OR MB 39831 OR MB39831 OR Temodar OR Temodal OR Schering-Plough brand of temozolomide OR Essex brand of temozolomide OR Schering brand of temozolomide OR TMZ-Bioshuttle OR CCRG 81045 OR CCRG-81045 OR NSC 362856 OR NSC-362856 OR 85622-93-1

2. For Gliomas the following MeSH headings and text words will be used:

diffuse intrinsic pontine glioma OR diffuse intrinsic pontine gliomas OR diffuse intrinsic pontine gliom* OR DIPG OR pontine gliom* OR diffuse pontine gliom* OR brainstem gliom* OR brain stem gliom* OR brainstem tumor* OR brainstem tumour* OR brain stem tumor* OR brain stem tumour* OR high grade glioma OR high grade gliomas OR high grade gliom* OR glioblastoma multiforme OR glioblastoma OR glioblastom* OR anaplastic astrocytoma OR fibrillary astrocytoma OR GBM OR diffuse astrocytoma OR diffuse fibrillary astrocytoma OR astrocytom*

3. For Children and adolescents the following MeSH headings and text words will be used:

infan* OR newborn* OR new-born* OR perinat* OR neonat* OR baby OR baby* OR babies OR toddler* OR minors OR minors* OR boy OR boys OR boyfriend OR boyhood OR girl* OR kid OR kids OR child OR child* OR children* OR schoolchild* OR schoolchild OR school child[tiab] OR school child*[tiab] OR adolescen* OR juvenil* OR youth* OR teen* OR under*age* OR pubescen* OR pediatrics[mh] OR pediatric* OR paediatric* OR peadiatric* OR school[tiab] OR school*[tiab] OR prematur* OR preterm* OR “young adult" OR “young adults" OR young adult [mh]

Final search 1 AND 2 AND 3

[tiab = title, abstract $; \mathrm{mh}=\mathrm{MeSH}$ term; ${ }^{*}=$ zero or more characters $]$

\section{Appendix 3. Search strategy for EMBASE (Ovid)}

1. For Temolozomide the following Emtree terms and text words will be used:

1. exp temozolomide/

2. temolozomide.mp.

3. (methazolastone or MB 39831 or MB-39831 or Temodar or Temodal).mp.

4. (TMZ-Bioshuttle or CCRG 81045 or CCRG-81045 or NSC 362856 or NSC-362856).mp.

5. 85622-93-1.rn.

6. or/1-5

2. For Gliomas the following Emtree terms and text words will be used:

1. exp pontine glioma/

2. (diffuse intrinsic pontine glioma or diffuse intrinsic pontine gliomas or diffuse intrinsic pontine gliom $\$$ ).mp.

3. (DIPG or pontine gliom $\$$ or diffuse pontine gliom $\$$ ). $\mathrm{mp}$.

4. (brainstem gliom $\$$ or brain stem gliom $\$$ ). $m p$.

5. (brainstem tumor $\$$ or brainstem tumour $\$$ or brain stem tumor $\$$ or brain stem tumour $\$$ ).mp.

6. (high grade glioma or high grade gliomas or high grade glioma $\$$ ).mp.

7. exp glioblastoma/

8. (glioblastoma multiforme or glioblastoma or glioblastom\$).mp.

9. anaplastic astrocytoma.mp.

10. exp astrocytoma/

11. (fibrillary astrocytoma or GBM or diffuse astrocytoma or diffuse fibrillary astrocytoma or astrocytom\$).mp.

12. or/1-11

3. For Children and adolescents the following Emtree terms and text words will be used:

1. infan\$.mp.

2. (newborn\$ or new-born\$).mp.

Temozolomide for children and adolescents with diffuse intrinsic pontine glioma (Protocol)

Copyright $\odot 2012$ The Cochrane Collaboration. Published by John Wiley \& Sons, Ltd. 
3. (perinat\$ or neonat\$).mp.

4. baby/

5. (baby or baby $\$$ or babies).mp.

6. toddler\$.mp.

7. (minors or minors $\$$ ).mp.

8. (boy or boys or boyfriend or boyhood).mp.

9. girl\$.mp.

10. (kid or kids).mp.

11. child/

12. (child or child $\$$ or children $\$$ ).mp.

13. school child/

14. (schoolchild\$ or schoolchild).mp.

15. (school child or school child\$).ti,ab.

16. (adolescen $\$$ or youth $\$$ or teen $\$$ ).mp.

17. (juvenil\$ or under\$age\$).mp.

18. pubescen $\$$.mp.

19. exp pediatrics/

20. (pediatric $\$$ or paediatric $\$$ or peadiatric $\$$ ).mp.

21. (school or school\$).mp.

22. (prematur\$ or preterm\$).mp.

23. (young adult or young adults or young adult\$).mp.

24. or/1-23

Final search: 1 and 2 and 3

$[\mathrm{mp}=$ title, abstract, subject headings, heading word, drug trade name, original title, device manufacturer, drug manufacturer name; ti,ab = title, abstract; / = Emtree term; $\$=$ zero or more characters; $r$ =CAS registry number]

\section{H I S T O R Y}

Protocol first published: Issue 10, 2012

\section{DECLARATIONSOF INTEREST}

None known.

\section{SOURCES OF SUPPORT}

\section{Internal sources}

- Dutch Cochrane Centre, Netherlands. 


\section{External sources}

- Stichting Kinderen Kankervrij (KiKa), Netherlands.

- Semmy Foundation, Netherlands.

- Egbers Foundation, Netherlands. 